\title{
Profiling of risk of subclinical atherosclerosis: possible interplay of genetic and environmental factors as the update of conventional approach
}

\author{
Igor A. Sobenin ${ }^{1,2}$, Veronika A. Myasoedova ${ }^{2}$, Tatiana V. Kirichenko, ${ }^{1,2}$, Varvara A. Orekhova ${ }^{1,3}$, Zukhra B. \\ Khasanova1, Vasily V. Sinyov ${ }^{1,2}$, Alexandra A. Melnichenko ${ }^{1,3}$, Andrey V. Grechko ${ }^{3}$, Alexander N. Orekhov ${ }^{2,4}$ \\ 'National Medical Research Center of Cardiology, Moscow 121552, Russian Federation. \\ ${ }^{2}$ Institute of General Pathology and Pathophysiology, Moscow 125315, Russian Federation. \\ ${ }^{3}$ Federal Scientific Clinical Center for Resuscitation and Rehabilitation, Moscow 109240, Russian Federation. \\ ${ }^{4}$ Institute for Atherosclerosis Research, Skolkovo Innovative Center, Skolkovo, Moscow 143025, Russian Federation.
}

Correspondence to: Dr. Igor A. Sobenin, Laboratory of Medical Genetics, National Medical Research Center of Cardiology, 15-a 3rd Cherepkovskaya Str, Moscow 121552, Russian Federation. E-mail: igor.sobenin@gmail.com

How to cite this article: Sobenin IA, Myasoedova VA, Kirichenko TV, Orekhova VA, Khasanova ZB, Sinyov VV, Melnichenko AA, Grechko AV, Orekhov AN. Profiling of risk of subclinical atherosclerosis: possible interplay of genetic and environmental factors as the update of conventional approach. Vessel P/us 2019;3:15. http://dx.doi.org/10.20517/2574-1209.2019.09

Received: 3 Dec 2018 First Decision: 29 Jan 2019 Revised: 14 Feb 2019 Accepted: 15 Feb 2019 Published: 6 May 2019

Science Editor: Alexander N. Orekhov Copy Editor: Cai-Hong Wang Production Editor: Huan-Liang Wu

\begin{abstract}
Aim: To explore whether geographical location, genetic and environmental factors are associated with carotid atherosclerosis in high-risk individuals.
\end{abstract}

Methods: In Moscow 470 apparently healthy, asymptomatic volunteer subjects with a high cardiovascular disease risk were recruited to participate in a cross-sectional study. Carotid intima-media thickness (cIMT), a validated biomarker for present and future cardiovascular disease risk, was assessed by means of high resolution ultrasound scans in subjects.

Results: The total burden of conventional cardiovascular risk factors explained $21 \%$ of the clMT variability; the mutational burden of mitochondrial genome defined by heteroplasmic mutations m.652delG, m.3256C>T, m.13513G>A, m.14459G $>A$, and m.15059G >A independently explained 23\% variability; the combination of conventional and genetic risk factors increased explanatory level to $36 \%$. Further exploratory statistical analyses showed air pollution as an independent risk factor for cIMT.

Conclusion: In our study we confirmed and expanded the existence of a European geographic gradient of atherosclerosis risk and its association with cardiovascular disease risk. Geographical, environmental (particularly, air pollution) - 
and genetic risk factors (particularly, mutant variants of mitochondrial genome) may interplay in the formation of susceptibility to atherosclerosis.

Keywords: Carotid atherosclerosis, high-risk patients, mitochondrial DNA mutations, air pollution, convenient cardiovascular risk factors

\section{INTRODUCTION}

Early detection and treatment of patients with a high risk of atherosclerosis is an urgent medical problem. Within this aspect, the identification of markers of subclinical atherosclerosis is an essential factor ${ }^{[1]}$. The thickness of the intima-media layer of the carotid arteries [carotid intima-media thickness (cIMT)], determined by high-resolution ultrasonography, is considered to be a validated and conventionally accepted non-invasive marker of subclinical atherosclerosis, that is used in clinical and epidemiological studies to assess the effect of traditional and new cardiovascular risk factors on atherosclerosis ${ }^{[2]}$. Since there is a correlation between cIMT and the degree of development of coronary atherosclerosis, and this factor has a prognostic significance in relation to the clinical manifestations of atherosclerosis, it is proposed as a surrogate marker of systemic atherosclerosis, including coronary one ${ }^{[3,4]}$. The classic cardiovascular risk factors are weakly associated with the cIMT of the carotid arteries, thus suggesting the presence of other factors that determine the risk of developing atherosclerosis. The results of a recent European multicenter study [The Carotid Intima Media Thickness (IMT) and IMT-Progression as Predictors of Vascular Events in a High Risk European Population Study, or IMPROVE Study] revealed the existence of a geographical gradient of the cIMT, coupled with a known gradient of cardiovascular mortality ${ }^{[5]}$. The detected southnorth geographic gradient of cIMT did not depend on interpopulation differences in the cumulative effects of conventional cardiovascular risk factors. It has been suggested that other mechanisms play a role in the origin of this gradient, including hereditary, socio-economic and environmental factors. In order to verify this assumption, we conducted our own population-based cross-sectional study in Moscow, the methodology of which could allow us to regard the results as a significant addition to the data from the European IMPROVE study. In particular, we aimed to explore whether geographical location, genetic and environmental factors are associated with carotid atherosclerosis in high-risk individuals, thus focusing on the possibility of specific interplay of genetic and environmental factors.

\section{METHODS}

\section{Patients}

The study was performed at municipal outpatient clinics in Moscow, Russia. The study was organized in accordance with international and domestic standards of quality clinical practice, namely, Helsinki Declaration of 1975 as revised in 1983, 2008 and 2013, and was approved by the local ethics committee. The study involved 470 subjects (200 men and 270 women) without clinical manifestations of atherosclerosis randomly recruited from the visitors' flow who have passed a routine screening for cardiovascular risk factors, as a screening subpart of Atherosclerosis Monitoring and Atherogenicity Reduction Study (ClinicalTrials.gov Identifier: NCT01734707) and Monocyte Activation in Preclinical Atherosclerosis Study (ClinicalTrials.gov Identifier: NCT02126280). The criteria for inclusion and exclusion were designed from those of IMPROVE Study, which allowed to form an observation group that is completely comparable with samples from European populations.

We have recruited men and women aged 55-79 years old who had at least three conventional risk factors for cardiovascular diseases. The latter included: hypercholesterolemia (low-density lipoprotein cholesterol level $>160 \mathrm{mg} / \mathrm{dL}$, or prescribed to cholesterol-lowering medications), hypertriglyceridemia (triglycerides > $200 \mathrm{mg} / \mathrm{dL}$, or prescribed to triglyceride-lowering drugs), low HDL-cholesterol (below $40 \mathrm{mg} / \mathrm{dL}$ ), arterial 
hypertension (diastolic blood pressure $>90 \mathrm{mmHg}$ and/or systolic blood pressure $>140 \mathrm{mmHg}$, or prescribed to antihypertensive drugs), the presence of diabetes mellitus or impaired glucose tolerance (blood sugar $>$ $110 \mathrm{mg} / \mathrm{dL}$, or prescribed to regular insulin injections and/or sugar-lowering drugs), smoking (consumption of $>10$ cigarettes per day for thirty preceding months), and the presence of family history of cardiovascular diseases. For women, an additional inclusion criterion was the presence of menopause (spontaneous or surgical) of more than 5-years duration.

Exclusion criteria were: abnormal anatomical configuration of the neck and neck muscles, severe tortuosity or unusual layout of the carotid arteries and its branches, the history of clinical manifestations of atherosclerosis (myocardial infarction, angina pectoris, acute and transient cerebral ischemic attacks, aortic aneurisms, revascularization of carotid, coronary or peripheral arteries), chronic heart failure of 3-4 functional class, and the presence of severe concomitant diseases.

Clinical examination of study participants included a biochemical analysis of serum lipid profile, identification of the main cardiovascular risk factors, calculating a prognostic 10-year risk of developing coronary heart disease, myocardial infarction and sudden coronary death ${ }^{[6]}$, and quantitative ultrasound diagnostics of carotid atherosclerosis.

\section{Lipid analysis}

Venous blood was taken after overnight fasting. To obtain serum, the blood was incubated for $1 \mathrm{~h}$ at $37^{\circ} \mathrm{C}$ and centrifuged for $15 \mathrm{~min}$ at $1,500 \mathrm{~g}$. Cholesterol and triglyceride levels were measured by commercial enzymatic kits (Cholesterol-32-Vital, and Triglycerides-22-Vital, respectively; Vital Diagnostics SPb, St. Petersburg, Russia). Serum HDL cholesterol concentrations were measured after precipitation with magnesium chloride phosphotungstic acid reagent (HDL-Cholesterol-04-Vital, Vital Diagnostics SPb, St. Petersburg, Russia). Serum LDL cholesterol was calculated by Friedewald formula as the difference between total cholesterol and the sum of HDL cholesterol and 1/5 triglycerides, and the ratio LDL-C/HDL-C was calculated.

\section{Ultrasonographic examination}

For diagnostics of carotid atherosclerosis, high-resolution B-mode carotid arterial ultrasonography imaging was used (SSI-6000 ultrasound system, SonoScape, China, equipped by 7.5-MHz L741 linear array probe). The protocol of ultrasound examination developed earlier by Salonen et al. ${ }^{[7]}$, was used. The cIMT measurements were carried out by a certified operator with M'Ath software package (IMT, France). The extent of carotid atherosclerosis were evaluated as described elsewhere ${ }^{[8,9]}$.

\section{MtDNA genotyping}

DNA was isolated from whole venous blood with commercial kit for DNA isolation and purification (QIAGEN GmbH, Germany). DNA concentration in samples was determined by NanoPhotometer Pearl UV/ Vis SDRAM P-34 (IMPLEN, Germany); the samples were kept in TE buffer at a concentration of $0.03 \mu \mathrm{g} / \mu \mathrm{L}$. Genotyping of mtDNA was performed for heteroplasmic mutations m.652 delG, m.1555A>G, m.3336T>C, m.3256C $>$ T, m.5178C $>$ A, m.12315G $>$ A, m.13513G $>A, m .14459 \mathrm{G}>\mathrm{A}, \mathrm{m} .14846 \mathrm{G}>\mathrm{A}$, and m.15059G $>\mathrm{A}$. For the amplification of mitochondrial DNA fragments, PCR method followed by pyrosequencing, with earlier described primers and conditions were used ${ }^{[9]}$. In brief, to quantitatively evaluate mutant allele, a method of pyrosequencing was adapted for the condition when normal and mutant alleles may be present in a biological specimen; the defective allele was quantified by analyzing the peak heights in the pyrogram of one-chained PCR-fragments of a mitochondrial genome ${ }^{[9-11]}$.

\section{Statistical analysis}

Data processing was performed using the SPSS software package, version 22.0 (IBM Corp., Chicago, IL, USA). Subprograms of descriptive statistics, variational analysis, parametric and nonparametric statistics, 
Table 1. Descriptive data on study participants

\begin{tabular}{lllll}
\hline Variable & Men, $\boldsymbol{n}=\mathbf{2 0 0}$ & Women, $\boldsymbol{n}=\mathbf{2 7 0}$ & $\boldsymbol{P}$ value & Total, $\boldsymbol{n}=\mathbf{4 7 0}$ \\
\hline Age, years & $61.4(59.9-62.5)$ & $66.1(65.5-67.1)$ & $<0.001$ & $64.1(63.6-65.1)$ \\
Body mass index, $\mathrm{kg} / \mathrm{m}^{2}$ & $27.0(26.5-27.5)$ & $27.8(27.3-28.3)$ & 0.033 & $27.5(27.1-27.9)$ \\
Systolic blood pressure, $\mathrm{mmHg}$ & $148(145-150)$ & $144(142-146)$ & 0.026 & $145(144-147)$ \\
Diastolic blood pressure, $\mathrm{mmHg}$ & $89(87-90)$ & $85(84-86)$ & $<0.001$ & $86(85-87)$ \\
Current smokers, \% & 15 & 12 & 0.4 & 13 \\
Never smokers, \% & 56 & 78 & $<0.001$ & 70 \\
Smoking cessation, \% & 29 & 10 & $<0.001$ & 17 \\
Smoking experience, years & $10.0(7.8-12.3)$ & $4.8(3.4-6.1)$ & $<0.001$ & $6.8(5.6-8.0)$ \\
Diabetes mellitus, \% & 8 & 5 & 0.20 & 6 \\
Arterial hypertension, \% & 76 & 77 & 0.80 & 77 \\
Menopause, years & - & $16.1(15.2-17.0)$ & - & - \\
Family history of: & 25 & & & 0.059 \\
$\quad$ Myocardial infarction & 35 & 33 & $<0.001$ & 30 \\
Arterial hypertension & 14 & 12 & 0.60 & 13 \\
Diabetes mellitus & $238(230-245)$ & $261(255-267)$ & $<0.001$ & $252(248-257)$ \\
Total cholesterol, mg/dL & $152(140-165)$ & $134(127-140)$ & 0.004 & $140(134-147)$ \\
Triglycerides, mg/dL & $60(58-62)$ & $70(69-72)$ & $<0.001$ & $66(65-68)$ \\
HDL cholesterol, mg/dL & $147(141-154)$ & $164(159-169)$ & $<0.001$ & $158(154-162)$ \\
LDL cholesterol, mg/dL & $2.6(2.4-2.7)$ & $2.5(2.4-2.6)$ & 0.20 & $2.5(2.4-2.6)$ \\
LDL/HDL ratio (Atherogenic index) & & & & \\
\hline
\end{tabular}

covariance analysis and linear regression were used. Data are presented as the mean and $95 \%$ confidence interval $^{[12]}$. The significance of differences was defined at the 0.05 level of confidence.

\section{RESULTS}

Clinical and anthropometric characteristics, and lipid profile of study participants are presented in Table 1.

As follows from the presented data, the cohort of study participants was, in general, at early retirement age, with overweight but not obesity, and with mild systolic arterial hypertension. The inclusion criteria used in this study predetermined a high proportion of participants with diagnosed hypertension (77\%). Accordingly, there was a high proportion of participants with a family history of myocardial infarction (30\%), hypertension (49\%) and diabetes (13\%). The proportion of subjects with diabetes (6\%) was comparable to that in population (reported occurrence, $4.5 \%-6 \%$ ). The share of smokers (13\%) was rather low, possibly due to the features of the surveyed contingent (older people, mostly with higher education, and to a certain extent focused on maintaining a healthy lifestyle).

Men and women differed by most of clinical and anthropometric parameters. Men were younger $(P<$ 0.001), had a lower body mass index $(P=0.033)$ presumably due to lower amount of abdominal fat, were characterized by higher blood pressure along with less likely family history of hypertension. The differences in family history of myocardial infarction and diabetes did not reach statistical significance.

Men had a higher proportion of smokers, the mean duration of smoking, and a lower proportion of neversmokers. At the same time, they also had a higher proportion of past-smokers, that also indicates the higher compliance of the surveyed population to the healthy lifestyle.

According to lipid measurements, moderate deviations in the lipid profile of the blood serum were observed. The presented mean values indicate the presence of moderate hypercholesterolemia with normal triglycerides. Elevated levels of total cholesterol were caused primarily by LDL cholesterol. At the same time, generally normal levels of HDL cholesterol were observed; as a result, LDL-C/HDL-C ratio remained within the normal range (the upper limit of normal values was determined as 3.0), thus indicating that balanced lipid metabolism was maintained. High levels of HDL cholesterol (taking the recommended lower 
Table 2. Ultrasound characteristics of carotid atherosclerosis

\begin{tabular}{|c|c|c|c|c|}
\hline Variable & Men, $n=\mathbf{2 0 0}$ & Women, $n=270$ & $P$ value & Total, $n=470$ \\
\hline Mean cIMT, mm & $0.819(0.798-0.840)$ & $0.827(0.813-0.842)$ & 0.5 & $0.824(0.812-0.836)$ \\
\hline Carotid artery internal diameter, $\mathrm{mm}$ & $7.66(7.55-7.78)$ & $7.24(7.17-7.31)$ & $<0.001$ & $7.40(7.34-7.47)$ \\
\hline
\end{tabular}

Table 3. Inter-population comparison of ultrasound characteristics of carotid atherosclerosis

\begin{tabular}{|c|c|c|c|}
\hline Population-derived sample & Mean cIMT, mm & Latitude & Longitude \\
\hline${ }^{\star}$ Perugia, Italy, $n=542$ & $0.70(0.69-0.71)$ & $43^{\circ}$ & $12^{\circ}$ \\
\hline${ }^{\star}$ Milan, Italy, $n=553$ & $0.72(0.71-0.73)$ & $45^{\circ}$ & $9^{\circ}$ \\
\hline${ }^{\star}$ Paris, France, $n=501$ & $0.68(0.67-0.69)$ & $48^{\circ}$ & $2^{\circ}$ \\
\hline${ }^{\star}$ Groningen, The Netherlands, $n=532$ & $0.72(0.71-0.73)$ & $53^{\circ}$ & $7^{\circ}$ \\
\hline *Stockholm, Sweden, $n=533$ & $0.79(0.78-0.80)$ & $59^{\circ}$ & $18^{\circ}$ \\
\hline${ }^{\star}$ Kuopio, Finland, $n=1,050$ & $0.76(0.75-0.77)$ & $62^{\circ}$ & $28^{\circ}$ \\
\hline Moscow, Russia, $n=472$ & $0.82(0.81-0.84)$ & $56^{\circ}$ & $38^{\circ}$ \\
\hline
\end{tabular}

${ }^{\star}$ The data are derived from published results of IMPROVE Study ${ }^{[5]}$

threshold of $50 \mathrm{mg} / \mathrm{dL}$ ) may also indirectly indicate the adherence to a healthy lifestyle, in particular, regular consumption of fresh vegetables and fruits.

Women differed significantly from men by all lipid parameters. They had higher cholesterol levels in both lipoprotein fractions (and as a result, in total cholesterol), and lower triglyceride levels. However, their LDL-C/HDL-C ratio remained normal, as in men.

The direct quantitative characteristics of atherosclerosis, resulted from ultrasound scanning of the carotid arteries followed by cIMT measurements, are provided in Table 2. In men, the diameter of the carotid arteries was significantly larger than in women $(P<0.001)$. However, there were no significant differences between men and women in the mean cIMT of the carotid arteries. The absence of such differences allowed us to carry out a regression analysis of the dependence of cIMT on conventional risk factors without taking into account sex differences. This analysis showed that the mean cIMT correlates with age $(P<$ $0.001)$, systolic blood pressure $(P<0.001)$, HDL cholesterol $(P=0.039)$ and LDL cholesterol $(P=0.003)$. The regression model was significant at $P<0.001$; the adjusted $R^{2}$ value was 0.209 .

The data on the direct quantitative characteristics of cIMT were compared with the results of the IMPROVE Study $^{[5]}$. The results of the comparison are presented in Table 3. Despite the fact that the inclusion criteria used in the study allowed us to form an observation group comparable to European samples for the cumulative effects of traditional cardiovascular risk factors, the mean cIMT in the high-risk patients from Moscow population was significantly higher than in any one from European populations at $P<0.001$.

Correlation analysis confirmed the existence of a geographic gradient of cIMT revealed in the IMPROVE Study. The correlation coefficient between the mean cIMT values and the geographical position of the research center, calculated as the geometric mean of geographical latitude and longitude, was $0.905(P=0.005)$.

The analysis of the relationship between the mean cIMT arteries and the standardized mortality from coronary heart disease, obtained from the WHO database (http://www.euro.who.int) was performed. The correlation coefficient between these variables was $0.853(P=0.015)$. The logarithmic model of this regression was reliable at $P=0.003$; the adjusted $R^{2}$ value was 0.860 .

We have also performed the linear regression analysis, where cIMT was taken as dependent variable, and convenient cardiovascular risk factors (age, sex, body mass index, systolic and diastolic blood pressure, 
smoking status, LDL cholesterol, HDL cholesterol, serum triglycerides, menopausal status for women, family history of myocardial infarction) as independent variables. It was found that the total burden of these risk factors explains $21 \%$ of the cIMT variability. Since conventional risk factors do not fully explain the variability of cIMT in the samples from European populations and the Moscow high-risk group, it should be assumed that other factors play a role in the formation of atherosclerosis predisposition.

One of these factors may be the adverse effects of the external environment. As a possible characteristic of the environmental situation, we used the mean annual integrated air pollution index. It was calculated as the sum of the ratios of the concentrations of the five major pollutants (nitrogen oxide, nitrogen dioxide, carbon monoxide, ozone and formaldehyde) to the maximum permissible concentration in the atmosphere. It was found that standardized mortality from coronary heart disease tends to correlate with the atmospheric pollution index: the correlation coefficient was 0.742 with $P=0.056$. At the same time, the air pollution index correlated with the mean cIMT: the correlation coefficient was 0.812 with $P=0.026$.

The other acting non-beneficial factor may be the genetic background. In our study, we have assessed the mutation burden of mitochondrial DNA by those heteroplasmic variants that have been previously shown to be associated with atherosclerotic lesions in human aorta and also with carotid atherosclerosis ${ }^{[9-11]}$. As it was shown in linear regression model, 5 of 10 mutations, for which the level of heteroplasmy was measured, independently provided the explanatory level of 23\% for cIMT variation by adjusted $R^{2}$ at $P<0.001$ (Fisher's exact test 11.21; $P<0.001$ ); these were m.652delG, m.3256C $>\mathrm{T}, \mathrm{m} .13513 \mathrm{G}>\mathrm{A}, \mathrm{m} .14459 \mathrm{G}>\mathrm{A}$, and $\mathrm{m} .15059 \mathrm{G}>\mathrm{A}$. The combined model, which included conventional risk factors and the above mutations provided significantly better explanatory level (36\%, Fisher's exact test 8.28; $P<0.001)$.

\section{DISCUSSION}

In this study, we used one of the generally accepted methods for assessing susceptibility to atherosclerosis, namely, non-invasive ultrasound scanning of the carotid arteries in high-resolution mode. This approach allowed to obtain direct quantitative estimate of the degree of development of subclinical atherosclerosis in individuals with a high risk of CHD. The criteria used for inclusion in the study allowed us to form a sample well comparable to the European samples in the IMPROVE Study on the cumulative effects of traditional cardiovascular risk factors. A direct comparison of the measurement of cIMT with European data showed that in spite of the same cumulative risk, the Moscow population-derived sample was more prone to atherosclerosis.

We were able to expand significantly the hypothesis on the existence of a geographic gradient of the variability of cIMT, and this gradient was associated not only with geographical latitude, as it was demonstrated in the IMPROVE Study, but also with geographical longitude. The association of the cIMT gradient with the gradient of cardiovascular mortality was confirmed, thus allowing to describe cIMT as the risk factor for atherosclerotic disease and its clinical manifestations.

It was confirmed that the variability of cIMT is not sufficiently explained by the cumulative effect of conventional risk factors: the explanatory nature of the used models for assessing the association of risk factors with cIMT was $21 \%$. Therefore, it was rather logical to assume that previously unexplored (or insufficiently studied) mechanisms of susceptibility to atherosclerosis may be of importance. The latter may include hereditary, socio-economic and environmental factors. The cross-sectional study in the sample from Moscow population, the methodology of which allowed it to be regarded as an addition to the data from the IMPROVE Study, showed that the ecological situation can be one of the factors associated with both the development of subclinical atherosclerosis and its clinical manifestations and complications.

The hypothesis on the possible role of the damage of mitochondrial DNA in atherosclerosis development has raised relatively recently but gains more and more experimental and clinical background. It is known that 
genetic factors evolved from variation of nuclear genome can be attributed approximately to $5 \%$ variability of atherosclerotic diseas ${ }^{[13]}$. On the opposite, the latest findings on the role of mitochondrial DNA variation in individual predisposition to atherosclerosis provide a growing body of evidence and the expectation of the breakthrough in understanding of molecular mechanisms and pathways of atherogenesis ${ }^{[14,15]}$. Heteroplasmic mtDNA mutations represent a promising molecular biomarker of genetic susceptibility to atherosclerosis and related pathologies ${ }^{[16-19]}$. In our study, we have demonstrated that several atherosclerosis-associated mutations of mitochondrial DNA taken together as a mutation burden of mitochondrial genome possess their own explanatory power and give a significant increase atop of assessment of conventional risk factors.

Undoubtedly, this study has certain limitations. First, the study was performed on a limited and fairly specific contingent (Muscovites, the elderly persons, mostly with higher education, focused on maintaining a healthy lifestyle, but with high level of convenient cardiovascular risk factors). Data from IMPROVE Study show that the cIMT varies significantly even between populations that are geographically close to each other. It is clear that in Russia in the areas differing not only geographically, but also in socio-economic, environmental and ethnic aspects, the variability of cIMT, as well as cardiovascular mortality, can differ significantly. The only possible resolution of this restriction may be the performance of similarly designed cross-sectional studies in various regions of Russia.

The second limitation is that the study did not assess socio-economic and ethnic variables. These unaccounted factors may also play a role in the formation of susceptibility to atherosclerosis and, undoubtedly, should be studied in further population-based studies.

The third limitation comes from the inability to quantitatively explore the interaction of mitochondrial DNA variants with conventional cardiovascular risk factors and environmental ones; therefore, the hypothesis on their interplay in the development of atherosclerosis remains the plausible idea, which needs further investigations. Besides, the fundamental problem still exists, since the molecular mechanisms whereby mitochondrial genome mutations may promote atherogenesis remain obscure. The most intriguing solution of this problem may be related to generation of cellular lines with edited mtDNA, which should reproduce pathologic phenotype and serve as the model for studying the effects of certain mtDNA mutations on cell functionality and mitochondrial dysfunction.

Finally, of all possible environmental factors theoretically capable of influencing the formation of phenotypic susceptibility to atherosclerosis (namely, diffuse intimal thickening, diagnosed ultrasonographically as an increased cIMT), only the integral indicator of atmospheric pollution was evaluated in our study. This indicator is neither standardized nor generally accepted. There is still no single worldwide database on air pollution. Each country uses its own standards, maximum permissible levels for the content of pollutants in the atmosphere, and methods for their determination. One of the adverse environmental factors is the dust load, which is not regulated and is not evaluated in the vast majority of countries around the world. In our study, the association of the cIMT with the content of dust particles in the atmosphere was not evaluated, mainly due to the lack of valid data. Therefore, the data on correlation between cIMT and air pollution index should be verified in a specially designed multicenter study; otherwise, these findings lead to rather speculative conclusions. However, a recent study in Germany showed that the content of solid dust particles of exhaust gases in atmospheric air is related to the degree of subclinical atherosclerosis, the prevalence of coronary heart disease and the incidence of myocardial infarction ${ }^{[20]}$.

Despite the above limitations, the results of our study suggest that environmental and genetic factors are involved in the formation of susceptibility to atherosclerosis and should be considered as plausible risk factors for cardiovascular diseases. However, the question on the plausibility of findings in routine clinical practice and preventive medicine remains disputable ${ }^{[21]}$. It is likely that early awareness on individual genetic predisposition to atherosclerosis may be the motivating factor for higher personal compliance to healthy 
lifestyle in more beneficial ecologic environment. On the other hand, it will also give a rise to earlier and more focused medicinal preventive measures.

\section{DECLARATIONS}

\section{Authors' contributions}

Concept of the study, general coordination and supervision of the research project, data analysis, statistical analysis, and draft manuscript writing: Sobenin IA

Patients' recruitment, clinical examination and clinical data acquisition: Myasoedova VA, Kirichenko TV, Orekhova VA, Grechko AV

MtDNA genotyping: Khasanova ZB, Sinyov VV, Melnichenko AA

Concept elaboration, general coordination and discussion: Orekhov AN

\section{Availability of data and materials}

Not applicable.

\section{Financial support and sponsorship}

The study was supported by the Russian Science Foundation (19-15-00297).

\section{Conflicts of interest}

All authors declared that there are no conflicts of interest.

\section{Ethics approval and consent to participate}

This study was kept in accordance with the Helsinki Declaration of 1975 as revised in 1983, 2008 and 2013. It was approved by the local ethics committee of the Institute for Atherosclerosis Research, Skolkovo Innovation Center, Moscow, Russia. All participants gave their written informed consent prior to their inclusion in the study.

\section{Consent for publication}

Not applicable.

\section{Copyright}

(c) The Author(s) 2019.

\section{REFERENCES}

1. Marcovina SM, Crea F, Davignon J, Kaski JC, Koenig W, et al. Biochemical and bioimaging markers for risk assessment and diagnosis in major cardiovascular diseases: a road to integration of complementary diagnostic tools. J Int Med 2007;261:214-34.

2. Stein JH, Korcarz CE, Hurst RT, Lonn E, Kendall CB, et al. American society of echocardiography carotid intima-media thickness task force. Use of carotid ultrasound to identify subclinical vascular disease and evaluate cardiovascular disease risk: a consensus statement from the American society of echocardiography carotid intima-media thickness task force. Endorsed by the society for vascular medicine. J Am Soc Echocardiogr 2008;21:93-111.

3. Amato M, Montrosi P, Ravani A, Oldani E, Galli S, et al. Carotid intima-media thickness by B-mode ultrasound as surrogate of coronary atherosclerosis: correlation with quantitative coronary angiography and coronary intravascular ultrasound findings. Eur Heart $\mathrm{J}$ 2007;28:2094-101.

4. Chambless LE, Heiss G, Folsom AR, Rosamond W, Szklo M, et al. Association of coronary heart disease incidence with carotid arterial wall thickness and major risk factors: the atherosclerosis risk in communities (ARIC) study, 1987-1993. Am J Epidemiol 1997;146:48394.

5. Baldassarre D, Nyyssönen K, Rauramaa R, de Faire U, Hamsten A, et al. IMPROVE study group. Cross-sectional analysis of baseline data to identify the major determinants of carotid intima-media thickness in a European population: the IMPROVE study. Eur Heart $\mathrm{J}$ 2010;31:614-22.

6. D'Agostino RB, Pencina MJ, Massaro JM, Coady S. Cardiovascular disease risk assessment: insights from Framingham. Glob Heart 2013;8:11-23.

7. Salonen R, Nyyssönen K, Porkkala E, Rummukainen J, Belder R, et al. Kuopio atherosclerosis prevention study (KAPS). A population- 
based primary preventive trial of the effect of LDL lowering on atherosclerotic progression in carotid and femoral arteries. Circulation 1995;92:1758-64.

8. Beux F, Carmassi S, Salvetti MV, Ghiadoni L, Huang Y, et al. Automatic evaluation of arterial diameter variation from vascular echographic images. Ultrasound Med Biol 2001;27:1621-9.

9. Sobenin IA, Sazonova MA, Postnov AY, Salonen JT, Bobryshev YV, et al. Association of mitochondrial genetic variation with carotid atherosclerosis. PLoS One 2013;8:e68070.

10. Sazonova M, Budnikov E, Khasanova Z, Sobenin I, Postnov A, et al. Studies of the human aortic intima by a direct quantitative assay of mutant alleles in the mitochondrial genome. Atherosclerosis 2009;204:184-90.

11. Sobenin IA, Sazonova MA, Postnov AY, Bobryshev YV, Orekhov AN. Mitochondrial mutations are associated with atherosclerotic lesions in the human aorta. Clin Dev Immunol 2012;2012:832464.

12. Lang TA, Secic M. How to report statistics in medicine: annotated guidelines for authors, editors, and reviewers. Second edition. American College of Physicians, Philadelphia, 2006.

13. Ioannidis JP. Prediction of cardiovascular disease outcomes and established cardiovascular risk factors by genome-wide association markers. Circ Cardiovasc Genet 2009;2:7-15.

14. Sobenin IA, Chistiakov DA, Bobryshev YV, Postnov AY, Orekhov AN. Mitochondrial mutations in atherosclerosis: new solutions in research and possible clinical applications. Curr Pharm Des 2013;19:5942-53.

15. Yu E, Mercer J, Bennett M. Mitochondria in vascular disease. Cardiovasc Res 2012;95:173-82.

16. Sobenin IA, Zhelankin AV, Mitrofanov KY, Sinyov VV, Sazonova MA, et al. Mutations of mitochondrial DNA in atherosclerosis and atherosclerosis-related diseases. Curr Pharm Des 2015;21:1158-63.

17. Sobenin IA, Zhelankin AV, Sinyov VV, Bobryshev YV, Orekhov AN. Mitochondrial aging: focus on mitochondrial DNA damage in atherosclerosis - A mini-review. Gerontology 2015;61:343-9.

18. Sinyov VV, Sazonova MA, Ryzhkova AI, Galitsyna EV, Melnichenko AA, et al. Potential use of buccal epithelium for genetic diagnosis of atherosclerosis using mtDNA mutations. Vessel Plus 2017;1:145-50.

19. Sazonova MA, Ryzhkova AI, Sinyov VV, Galitsyna EV, Orekhova VA, et al. New markers of atherosclerosis: a threshold level of heteroplasmy in mtDNA mutations. Vessel Plus 2017;1:182-91.

20. Hoffmann B, Moebus S, Dragano N, Möhlenkamp S, Memmesheimer M, et al. Heinz nixdorf recall investigative group. Residential traffic exposure and coronary heart disease: results from the Heinz Nixdorf Recall Study. Biomarkers 2009;14:74-8.

21. Orekhov AN, Ivanova EA. Introduction of the special issue "atherosclerosis and related diseases". Vessel Plus 2017;1:163-5. 